\title{
Extrapulmonary Tuberculosis Mimics Diffuse Metastatic Disease: A Case Report
}

\author{
Gulgun Engin ${ }^{1}$ Kızıldag Yirgin ${ }^{1}$ Hulya Kandemir ${ }^{2}$ \\ ${ }^{1}$ Department of Radiology, Oncology Institute, Istanbul University, \\ Istanbul,Turkey \\ 2 Department of Obstetrics and Gynecology, Istanbul University, \\ Istanbul Faculty of Medicine, Istanbul, Capa, Turkey \\ Indian J Radiol Imaging 2021;31:1039-1042.
}

\begin{abstract}
Address for correspondence Kizildag Yirgin, MD, Department of Radiology, Oncology Institute, Istanbul University, Istanbul, Turkey (e-mail: incikizil@hotmail.com).
\end{abstract}

\begin{abstract}
Background Extrapulmonary tuberculosis (TB) is associated with major diagnostic difficulties as it has many atypical symptoms and its imaging findings are similar to those of many other diseases.

Case Report We present the case of a 50-year-old woman with a complex cystic mass in her pelvis and a 2-month history of pain. Thoracoabdominal computed tomography (CT) revealed the following: (1) multiple supraclavicular, axillary, mediastinal, and abdominal pathologic lymph nodes with central hypoattenuation; (2) sternal and dorsal vertebral bone destruction with a soft tissue mass; and (3) multiple hypoattenuating masses in the liver. ${ }^{18} \mathrm{~F}$-fludeoxyglucose positron emission tomography/ computed tomography $\left({ }^{18} \mathrm{~F}-\mathrm{FDG} \mathrm{PET} / \mathrm{CT}\right)$ revealed pathologic uptake in the defined

Keywords

- computed tomography

- extrapulmonary tuberculosis

- imaging

- PET

- tuberculosis areas, which were similar to metastases. Chest X-ray was normal. Diffuse metastatic disease was suspected clinically, but careful analysis of the imaging findings and the final pathologic results revealed multisystemic extrapulmonary TB. Clinical and radiological recovery was significant after anti-TB treatment at the 6-month follow-up. Conclusion Extrapulmonary TB without pulmonary involvement is associated with major diagnostic difficulties, especially with PET-CT. However, recognition and understanding of the CT or MR imaging spectrum can aid in the correct diagnosis and management of the disease.
\end{abstract}

\section{Introduction}

Tuberculosis (TB) is the leading cause of death from infectious disease worldwide. TB can often be controlled with early and accurate detection and diagnosis. ${ }^{1}$ However, in cases without pulmonary TB, extrapulmonary TB is associated with major diagnostic difficulties because of its metastatic disease-like imaging findings and elevated levels of serum cancer antigen (CA) $125^{2,3}$ Recognition and understanding of the spectrum of extrapulmonary TBs imaging features can improve its early diagnosis and management.

Herein, we present a case of multisystemic extrapulmonary $\mathrm{TB}$ without pulmonary $\mathrm{TB}$ mimicking diffuse metastatic disease, and we discuss the diagnostic and differential criteria of this case. published online

December 13, 2021
DOI https://doi.org/

10.1055/s-0041-1739378.

ISSN 0971-3026. (c) 2021. Indian Radiological Association. All rights reserved.

This is an open access article published by Thieme under the terms of the Creative Commons Attribution-NonDerivative-NonCommercial-License, permitting copying and reproduction so long as the original work is given appropriate credit. Contents may not be used for commercial purposes, or adapted, remixed, transformed or built upon. (https://creativecommons.org/ licenses/by-nc-nd/4.0/)

Thieme Medical and Scientific Publishers Pvt. Ltd., A-12, 2nd Floor, Sector 2, Noida-201301 UP, India 


\section{Case Report}

We present the case of a 50-year-old woman with a 2-month history of pelvic pain. A vaginal examination revealed a left adnexal mass with tenderness on palpation. Transvaginal ultrasound revealed a complex cystic mass $(5 \times 4 \mathrm{~cm}$ in diameter) and a small amount of ascites in the pouch of Douglas. She had a high erythrocyte sedimentation rate $(113 \mathrm{~mm} / \mathrm{h}$; reference range $0-30 \mathrm{~mm} / \mathrm{h})$, but normal peripheral blood laboratory tests (including a complete blood cell count, routine serum chemistry, and C-reactive protein level). She did not have leukocytosis or monocytosis. She had high levels of the tumor markers carcinoembryonic antigen $(6.4 \mathrm{ng} / \mathrm{mL})$ and CA $125(40.65 \mathrm{U} / \mathrm{mL})$, whereas her levels of CA 15 to $3(22.9 \mathrm{U} / \mathrm{mL})$ and CA 19 to $9(24.65 \mathrm{U} / \mathrm{mL})$ were normal. Human immunodeficiency virus serology and sputum culture were negative, and no pathologic finding was evident on plain radiography of the chest and abdomen.

A decision was made to follow up with the patient, as she had been diagnosed with Leriche syndrome and had a history of multiple abdominal surgical interventions because of aortobifemoral bypass and graft complications. Six months later, thoracoabdominal computed tomography (CT) revealed multiple supraclavicular, axillary, mediastinal, and abdominal pathologic lymph nodes (1-4 cm in length) along the short axis with central hypoattenuation. We also observed bone destruction accompanied by a hypoattenuating soft tissue mass in the sternum and dorsal vertebrae, as well as multiple hypodense and minimally rim-enhancing liver lesions (1-2 cm in diameter). The lesion in the liver's left lobe had extended through the peritoneum and into the anterior abdominal wall ( - Fig. 1). Pelvic CT demonstrated a left adnexal complex cystic mass $(2 \times 4 \mathrm{~cm}$ in size) with thick septa and solid portions. ${ }^{18} \mathrm{~F}$-fludeoxyglucose positron emission tomography $\left({ }^{18} \mathrm{~F}-\mathrm{FDG}\right.$ PET)/CT revealed pathologic uptake in the disease areas, but not in the ovarian cystic lesion.

The patient had no pleural or peritoneal ascites. However, histopathologic analyses of ultrasound-guided core biopsies of the supraclavicular lymph node revealed granulomatous inflammatory reaction with necrosis, whereas liver biopsy revealed diffuse granulomatous inflammation and fibrosis. Histopathology of the sternal bone biopsy also revealed a granulomatous focus.

On the basis of the aforementioned findings, multisystemic extrapulmonary TB with a tubo-ovarian abscess was considered. The patient was started on anti-TB treatment consisting of isoniazid, rifampicin, ethambutol, and pyrazinamide for 2 months, which should have been followed by isoniazid and rifampicin for 9 months. However, her treatment was terminated after 2 months because of elevated liver enzymes.

At the 6-month follow-up, the patient's clinical recovery was marked by significant decreases in general body pain and palpable supraclavicular lymph nodes. The erythrocyte sedimentation rate remained high $(31 \mathrm{~mm} / \mathrm{h}$; reference value $0-20 \mathrm{~mm} / \mathrm{h}$ ), but was significantly decreased compared with the pretreatment value. Peripheral blood laboratory tests as performed pretreatment were within normal limits.
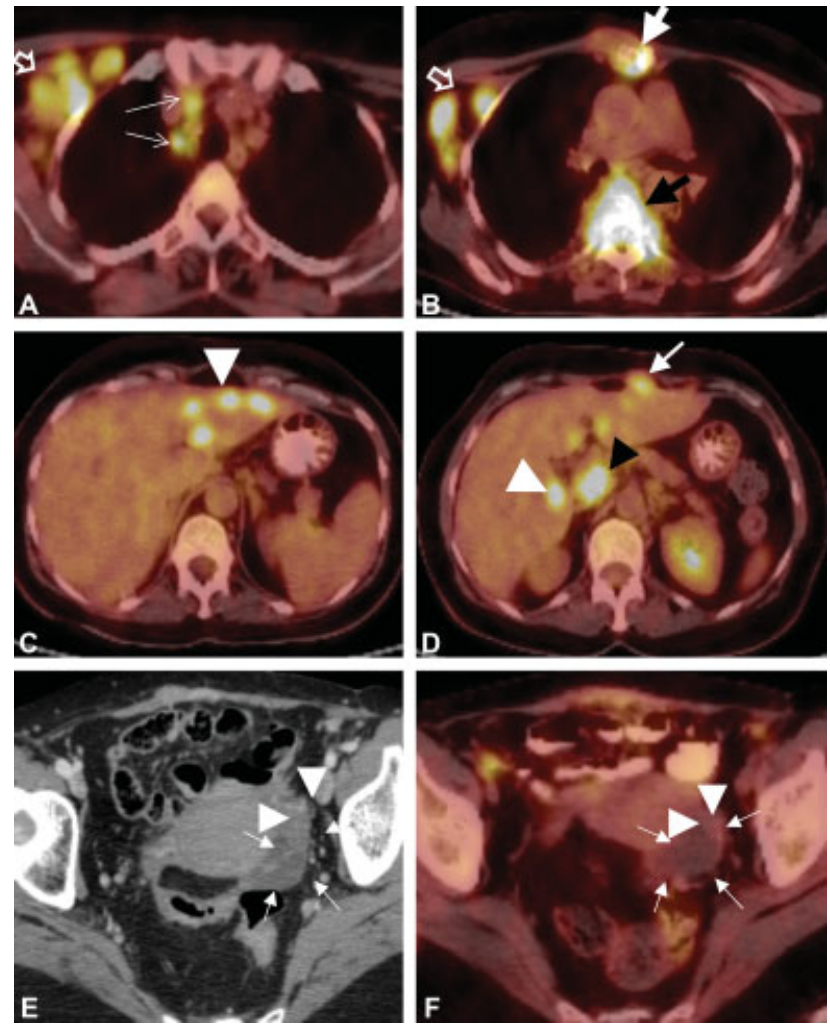

Fig. $1{ }^{18} \mathrm{~F}$-fludeoxyglucose positron emission tomography/ computed tomography ( ${ }^{18} \mathrm{~F}$-FDG PET/CT) shows false-positive metastatic disease findings in a case with multisystemic extrapulmonary tuberculosis. (A, B) Pathologic FDG uptake is seen in the supraclavicular (open arrows) and mediastinal lymph nodes (thin arrows) as well as in the sternal (thick white arrow) and dorsal vertebral (thick black arrow) bones with surrounding soft tissue masses. (C, D) ${ }^{18}$ F-FDG PET/CT shows pathologic uptake in the liver (white arrowheads) and peripancreatic lymph node (black arrowhead). An abscess in the left liver lobe extends through the peritoneum into the anterior abdominal wall (arrow). (E, F) Pelvic CT demonstrates a left adnexal complex cystic mass $2 \times 4 \mathrm{~cm}$ in diameter (arrows) with thick septa and solid portions (arrowheads)

corresponding to a tubo-ovarian abscess with pseudotumor papillary areas. ${ }^{18}$ F-FDG PET/CT does not reveal pathologic uptake in the cyst (arrows) or in its pseudotumor papillary areas (arrowheads).

Follow-up thoracoabdominal CT revealed decreases in the size of the Pott's abscesses as well as in the number and size of the lymph nodes and liver abscesses. These findings were compatible with a partial response to the anti-TB therapy.

\section{Discussion}

In this report, abdominal CT and ${ }^{18} \mathrm{~F}$-FDG PET/CT findings initially led to the suspicion of ovarian malignancy and diffuse metastatic disease, but careful analysis of CT imaging findings and pathologic results eventually revealed multisystemic extrapulmonary TB.

After the lymphatic system, the genitourinary system is the second-most frequent location of extrapulmonary TB. The fallopian tubes are involved in 90 to $100 \%$ cases of genital TB. ${ }^{4}$ Tubo-ovarian abscess is revealed as a multilocular cystic pelvic mass with pseudotumor areas that mimic solid portions of ovarian malignancy. CA 125 is a tumor marker of 
nonmucinous ovarian cancer that may also be increased in peritoneal TB. ${ }^{3}{ }^{18} \mathrm{~F}$-FDG PET/CT findings are not helpful in distinguishing this type of TB from ovarian malignancy.

A complex tubo-ovarian abscess may mimic the PET/CT imaging characteristics of an ovarian or pelvic malignancy. ${ }^{5}$ On PET/CT, abscesses often exhibit a hypometabolic center surrounded by an intensely FDG-avid rim. ${ }^{6,7}$ However, they can also exhibit solid and uniformly intense FDG uptake throughout. ${ }^{5,8}$ In their study, Sharma et al reported the results of 17 patients with proven tubercular tubo-ovarian masses who underwent ${ }^{18} \mathrm{~F}$-FDG-PET/CT imaging, and these findings were compared with those of ultrasound (for all patients), magnetic resonance imaging (MRI; for 9 patients), CT (for 4 patients), and laparotomy or laparoscopic findings (for 14 patients). The detection rates of tubo-ovarian masses with ${ }^{18} \mathrm{~F}$-FDG-PET/CT were similar to those obtained with CT or MRI, but there was less characterization of adnexal masses. However, ${ }^{18}$ F-FDG-PET/CT was equally as accurate as laparoscopy or laparotomy in detecting the presence, laterality, and activity of tubo-ovarian masses. The authors of the study concluded that ${ }^{18}$ F-FDG-PET/CT is clinically useful for noninvasively detecting the presence and activity of tubo-ovarian masses, but not for their characterization. ${ }^{9}$ In our case, there was no pathologic uptake in the ovarian cystic lesion on ${ }^{18} \mathrm{~F}$-FDG PET)/CT, which may have been due to inactivity.

Differential diagnosis becomes more difficult when extrapulmonary TB is a multisystemic disease, as in the case presented herein. However, knowing the characteristics, but not specific, imaging findings may facilitate the diagnosis.

TB lymphadenopathy is a common form of extrapulmonary TB. The cervical (63\%), mediastinal (27\%), and axillary (8-10\%) lymph nodes are the most commonly affected. Most cases are unilateral, including the patient described herein who had involvement of the supraclavicular, axillary, and mediastinal lymph nodes on the right. Peripheral enhanced and central necrotic lymph nodes are highly suggestive, but not pathognomonic, of TB. ${ }^{10}$ However, they should be differentiated from metastatic head and neck squamous cell carcinomas and inflammatory conditions. ${ }^{11}$ Nodal calcification often develops late in TB, which helps to differentiate TB nodes from malignancy.

Hepatic TB is an unusual form of extrapulmonary TB that constitutes less than $1 \%$ of all TB cases. The imaging findings of hepatic TB are nonspecific. ${ }^{12}$ Both micro- and macronodular forms can be observed, although macronodular forms are less common. The macronodular forms appear as hypoattenuating lesions 1 to $3 \mathrm{~cm}$ in diameter, or as a single mass with hepatosplenomegaly. ${ }^{13}$ MRI reveals hypointense and minimally enhanced honeycomb-like lesions on T1-weighted images. ${ }^{10}$ The differential diagnosis of the micronodular form includes metastasis, fungal infection, sarcoidosis, and lymphoma, whereas the differential diagnosis of the macronodular form includes metastasis, primary malignant tumor, or pyogenic abscess. Similar to metastatic disease, these lesions also show avid FDG uptake on PET/CT imaging. ${ }^{13}$ However, a liver abscess that extends through the peritoneum and into the extraperitoneal compartment suggests TB, as in the case presented herein. ${ }^{10}$

Lymphadenopathy is the most common manifestation of abdominal TB, which most commonly affects the mesenteric and peripancreatic groups. ${ }^{13}$ In general, the nodes are typically multiple and large, averaging 2 to $3 \mathrm{~cm}$ in diameter with a hypoattenuating center with or without calcification. In ${ }^{18}$ F-FDG PET/CT imaging, TB can lead to a false-positive metastatic lymph node diagnosis, as in our current case. ${ }^{14}$

Approximately, 1 to $3 \%$ of patients with TB have skeletal involvement. Skeletal TB most commonly involves the spine or weight-bearing joints, whereas the sternum is involved in $\sim 1 \%$ of cases. ${ }^{10}$ More than one vertebral body is typically affected. Paraspinal infection may involve the psoas muscle, resulting in Pott's disease. Calcification within the abscess is virtually pathognomonic of TB. ${ }^{4}$

The imaging findings of multisystemic extrapulmonary TB mimic metastatic diseases. Therefore, an understanding of the spectrum of imaging findings of extrapulmonary TB plays a crucial role in its early and accurate identification. However, PET/CT imaging findings have potentially serious pitfalls in the evaluation of extrapulmonary TB. It is important for those reading PET/CT results to be aware of this benign differential diagnosis of extrapulmonary multisystemic TB, even in the context of CT or MRI findings suspicious for malignancy.

\section{Informed Consent}

Written informed consent was obtained.

\section{Financial Support and Sponsorship}

Nil.

\section{Conflicts of Interest}

There are no conflicts of interest.

\section{Acknowledgments}

We would like to acknowledge www.makaletercume.com for their outstanding scientific proofreading and editing services that was provided for this manuscript.

\section{References}

1 Walzl G, McNerney R, du Plessis N, et al. Tuberculosis: advances and challenges in development of new diagnostics and biomarkers. Lancet Infect Dis 2018;18(07):e199-e210

2 Aisenberg GM, Jacobson K, Chemaly RF, Rolston KV, Raad II, Safdar A. Extrapulmonary tuberculosis active infection misdiagnosed as cancer: Mycobacterium tuberculosis disease in patients at a Comprehensive Cancer Center (2001-2005). Cancer 2005;104 (12):2882-2887

3 Liu Q Zhang Q Guan Q Xu JF, Shi QL. Abdominopelvic tuberculosis mimicking advanced ovarian cancer and pelvic inflammatory disease: a series of 28 female cases. Arch Gynecol Obstet 2014;289 (03):623-629

4 Uzunkoy A, Harma M, Harma M. Diagnosis of abdominal tuberculosis: experience from 11 cases and review of the literature. World J Gastroenterol 2004;10(24):3647-3649

5 Rakheja R, Makis W, Hickeson M. Bilateral tubo-ovarian abscess mimics ovarian cancer on MRI and (18)F-FDG PET/CT. Nucl Med Mol Imaging 2011;45(03):223-228 
6 Ichiya Y, Kuwabara Y, Sasaki M, et al. FDG-PET in infectious lesions: the detection and assessment of lesion activity. Ann Nucl Med 1996;10(02):185-191

7 Shreve PD, Anzai Y, Wahl RL. Pitfalls in oncologic diagnosis with FDG PET imaging: physiologic and benign variants. Radiographics 1999;19(01):61-77, quiz 150-151

8 Bleeker-Rovers CP, Warris A, Drenth JP, Corstens FH, Oyen WJ, Kullberg BJ. Diagnosis of Candida lung abscesses by $18 \mathrm{~F}-$ fluorodeoxyglucose positron emission tomography. Clin Microbiol Infect 2005;11(06):493-495

9 Sharma JB, Karmakar D, Kumar R, et al. Comparison of PET/CT with other imaging modalities in women with genital tuberculosis. Int J Gynaecol Obstet 2012;118(02):123-128
10 Engin G, Acunaș B, Acunaş G, Tunaci M. Imaging of extrapulmonary tuberculosis. Radiographics 2000;20(02):471-488, quiz 529-530, 532

11 Burrill J, Williams CJ, Bain G, Conder G, Hine AL, Misra RR. Tuberculosis: a radiologic review. Radiographics 2007;27(05): 1255-1273

12 Wang X, Shi X, Yi C, Chen Z, Zhang B, Zhang X. Hepatic tuberculosis mimics metastasis revealed by $18 \mathrm{~F}-\mathrm{FDG}$ PET/CT. Clin Nucl Med 2014;39(06):e325-e327

13 Leder RA, Low VH. Tuberculosis of the abdomen. Radiol Clin North Am 1995;33(04):691-705

14 Bozan N, Sakin YF, Parlak M, Bozkuş F Suppurative cervical tuberculous lymphadenitis mimicking a metastatic neck mass. J Craniofac Surg 2016;27(06):e565-e567 